\title{
Investigating the Vela SNR's Emission of Electron Cosmic Rays with CALET at the International Space Station
}

\section{Holger Motz ${ }^{a, *}$ on behalf of the CALET Collaboration}

(a complete list of authors can be found at the end of the proceedings)

${ }^{a}$ Waseda University, Faculty of Science and Engineering, Global Center for Science and Engineering Okubo 3-4-1, Shinjuku, Tokyo, Japan

E-mail: motz@aoni.waseda.jp

The ISS-based Calorimetric Electron Telescope (CALET) is directly measuring the energy spectrum of electron+positron cosmic rays up to $20 \mathrm{TeV}$. Cosmic-ray electrons of $\mathrm{TeV}$ region energy are limited by energy loss to a propagation range of about $1 \mathrm{kpc}$, therefore the expected sources are a few nearby supernova remnants (SNR), with the Vela SNR dominating the spectrum [1]. The latest spectrum measured by CALET [2] in combination with the positron-only flux published by AMS-02 [3] is fitted with a comprehensive model including nearby pulsars as the source of the positron excess. This model is extended to the TeV region by addition of the flux from the Vela SNR as calculated with DRAGON, with the integrated energy emitted in electron cosmic rays by the SNR as a variable scale factor. Exploring various scenarios for the time and energy dependence of the cosmic-ray release from Vela, under varied propagation conditions, best-fitting interpretations of the spectrum and upper limits on the emission of cosmic-ray electrons by Vela have been derived.

$37^{\text {th }}$ International Cosmic Ray Conference (ICRC 2021)

July 12 th - 23rd, 2021

Online - Berlin, Germany

\footnotetext{
${ }^{*}$ Presenter
} 


\section{Introduction}

It is widely accepted that SNR are the dominant source of both nuclei and electron cosmic rays, based on the agreement for the power law shape of the spectrum and the energy requirement to maintain the galactic cosmic-ray density. And while indirect measurements through gamma rays support the acceleration of cosmic rays in SNR, no direct measurement of cosmic rays from an individual source could be accomplished, due to their origin being masked by the random walk propagation in the galactic magnetic field. Cosmic-ray electrons of $\mathrm{TeV}$ region energy however are limited by energy loss to a propagation range of about $1 \mathrm{kpc}$. Therefore, the $\mathrm{TeV}$ region electron flux is a direct probe for acceleration of cosmic rays in the few nearby SNR which are possible sources. Ref. [1] established the most important candidate sources of electron cosmic rays at $\mathrm{TeV}$ energy: The Vela SNR is expected to dominate the spectrum in the TeV region due to its distance of $\sim 0.3 \mathrm{kpc}$ and age of $\sim 11 \mathrm{kyr}$. Significant contributions are expected from the Monogem SNR at also $\sim 0.3 \mathrm{kpc}$ distance but with an age of $\sim 86 \mathrm{kyr}$ at the lower end of the $\mathrm{TeV}$ region, and from Cygnus Loop with a larger distance of $\sim 0.44 \mathrm{kpc}$ and $\sim 20 \mathrm{kyr}$, giving a subdominant contribution in a similar energy range as Vela. As normalization of the spectra, the total energy emitted in electron cosmic rays above $1 \mathrm{GeV}$ is estimated to be $Q_{0_{S N R}} \sim 1 \times 10^{48} \mathrm{erg}$. Assuming generic continuous emission and delayed burst-like emission scenarios, the preferred range for $Q_{0_{S N R}}$, and upper limits on it, are derived in this study based on the latest all-electron spectrum measured by CALET. Secondary particles and the source of the positron excess comprise a well defined background contribution based the positron-only flux measured by AMS-02, which is thus used in a combined fitting with the CALET data.

\section{Methods}

\subsection{Propagation Models}

The nearby SNR spectra are calculated with the numerical propagation code DRAGON [4], which is also used to define the propagation parameters via calculation of the nuclei spectra, concurrently providing spectra of the secondary electrons and positrons forming part of the background.The propagation models explain the spectral hardening observed in the nuclei spectra by the slope of the diffusion coefficient with rigidity decreasing gradually from $\delta_{l}=0.5$ (Kraichnan). This allows for the common injection spectrum for all primary nuclei species to be a single-index $\left(\gamma_{i}\right)$ power law, with exponential cut off at rigidity $R_{\text {cut }}$ to model the spectra's subsequent softening. Measurements of the proton spectrum and the boron-to-carbon ratio together constrain the fundamental propagation parameters. Introducing a spatial dependence of the diffusion coefficient allows for good agreement of the model with measurement data. The diffusion coefficient increases exponentially with galactic radius $r$ for $r>r_{n}$, while constant for $r<r_{n}$, and with distance from the galactic plane $z$ for $z>z_{n}$, while constant for $z<z_{n}$. With $r_{n}=2 \mathrm{kpc}$ and $z_{n}=0.15 \mathrm{kpc}$, the regions of constant diffusion correspond to the galactic bulge and the thin disk respectively. Combining this spatial dependence with the rigidity dependence, the diffusion coefficient is expressed as

$$
D(r, z, R)=D_{0} \max \left\{e^{\left(r-r_{n}\right) / r_{s}}, 1\right\} \max \left\{e^{\left(z-z_{n}\right) / z_{s}}, 1\right\}\left(\frac{R}{R_{0}}\right)^{\delta_{l}}\left(1+\left(\frac{R}{R_{b}}\right)^{\frac{\delta_{l}-\delta_{h}}{s}}\right)^{-s} .
$$




\begin{tabular}{|c|c|c|c|c|}
\hline Model & $\mathrm{A}$ & $\mathrm{B}$ & $\mathrm{C}$ & $\mathrm{X}$ \\
\hline$D_{0}\left[10^{28} \mathrm{~cm}^{2} / \mathrm{s}\right]$ & 0.66 & 1.32 & 1.78 & 0.16 \\
$r_{s}[\mathrm{kpc}]$ & 4.5 & 4.5 & 4.5 & 3.0 \\
$L[\mathrm{kpc}]$ & 3 & 6 & 9 & 6 \\
$z_{s}[\mathrm{kpc}]$ & 1.8 & 3.5 & 5.3 & 0.6 \\
$R_{b}[\mathrm{TV}]$ & 0.5 & 0.5 & 0.5 & 1.0 \\
$\delta_{h}$ & 0.2 & 0.2 & 0.2 & 0.01 \\
$s$ & 0.05 & 0.05 & 0.05 & 0.3 \\
$v_{a}[\mathrm{~km} / \mathrm{s}]$ & 0 & 0 & 0 & 18 \\
\hline
\end{tabular}

\begin{tabular}{|c|c|c|c|c|}
\hline Marameter & Model & B & C & X \\
\hline$\gamma_{i}$ & 2.34 & 2.34 & 2.34 & 2.335 \\
$R_{\text {cut }}[\mathrm{TV}]$ & 100 & 100 & 100 & 30 \\
\hline & & & & \\
$D_{0(\text { sol })}\left[10^{28} \mathrm{~cm}^{2} / \mathrm{s}\right]$ & 2.68 & 5.35 & 7.22 & 1.31 \\
$\Phi_{0}[\mathrm{GV}]$ & 0.32 & 0.34 & 0.36 & 0.89 \\
$\Phi_{1}[\mathrm{GV}]$ & 2.0 & 2.0 & 2.0 & 0.37 \\
$\Phi(10 \mathrm{GV})[\mathrm{GV}]$ & 0.43 & 0.45 & 0.47 & 0.91 \\
& & & & \\
\hline
\end{tabular}

Table 1: Propagation model parameters: Diffusion condition parameters are listed in the left table, the injection spectrum parameters in the right table, top section, and derived parameters in the bottom section.

For comparison with the measurements, the DRAGON output is fitted to AMS-02 proton and B/C ratio data above $5 \mathrm{GeV} / \mathrm{n}$ with variable parameters to adjust flux normalization and solar modulation. Solar modulation is treated by a rigidity dependent potential based on Ref. [5] given by

$$
\Phi(R)=\Phi_{0}+\Phi_{1} \frac{1+\left(R / R_{r}\right)^{2}}{\left(R / R_{r}\right)^{3}}
$$

with $\Phi_{0}$ being a fully free parameter, while the reference rigidity $R_{r}$ is set to $0.5 \mathrm{GV}$ and $\Phi_{1}$ is varied in the interval [0,2] GV to remain in the range of rigidity dependence considered in Ref. [5].

Four specific propagation models are used in this study, designated A, B, C and X, for which the parameters are given in Table 1. Models $\mathrm{A}, \mathrm{B}$ and $\mathrm{C}$ reproduce the proton and $\mathrm{B} / \mathrm{C}$ ratio, with diffusion zone height and diffusion coefficient increasing from A to C. Model X simultaneously reproduces the helium spectrum measurements based on the same injection spectrum within experimental uncertainty, achieved by a stronger spatial variance of the diffusion coefficient in combination with diffusive re-acceleration, and by adjusting the break in the rigidity dependence of the diffusion coefficient for a balance between proton and helium spectra characteristics. The comparison between the results of the DRAGON nuclei spectra calculations and measured data is shown in Figure 1. The fit to AMS-02 proton and B/C (and helium for Model X) data yields reduced $\chi^{2}$ below one for all models.

\subsection{Electron and Positron Flux Parametrization}

Four components are considered in the modeling of the electron and positron spectra: Primary electrons from distant SNR, secondary electrons and positrons, pulsars as the source of the positron excess, and the electron flux from the nearby SNR. As this study is about generic cases of astrophysical sources, the pulsar explanation of the positron excess is chosen over non-standard dark matter and secondary production scenarios. The electron flux parameterization is written as

$$
\Phi_{e}^{-}=C_{e} E^{-\left(\gamma_{e}-\Delta \gamma_{e}\right)}\left(1+\left(\frac{E}{E_{b}}\right)^{\frac{\Delta \gamma_{e}}{s}}\right)^{s} e^{-\left(\frac{E}{E_{\text {cut }_{d}}}\right)}+\frac{C_{s}}{C_{n o r m}} \Phi_{s\left(e^{-}\right)}+\Phi_{\text {pulsars }}+\Phi_{\text {nearSNR }},
$$

and the positron spectrum as

$$
\Phi_{e}^{+}=\frac{C_{s}}{C_{n o r m}} \Phi_{s\left(e^{+}\right)}+\Phi_{\text {pulsars }} .
$$



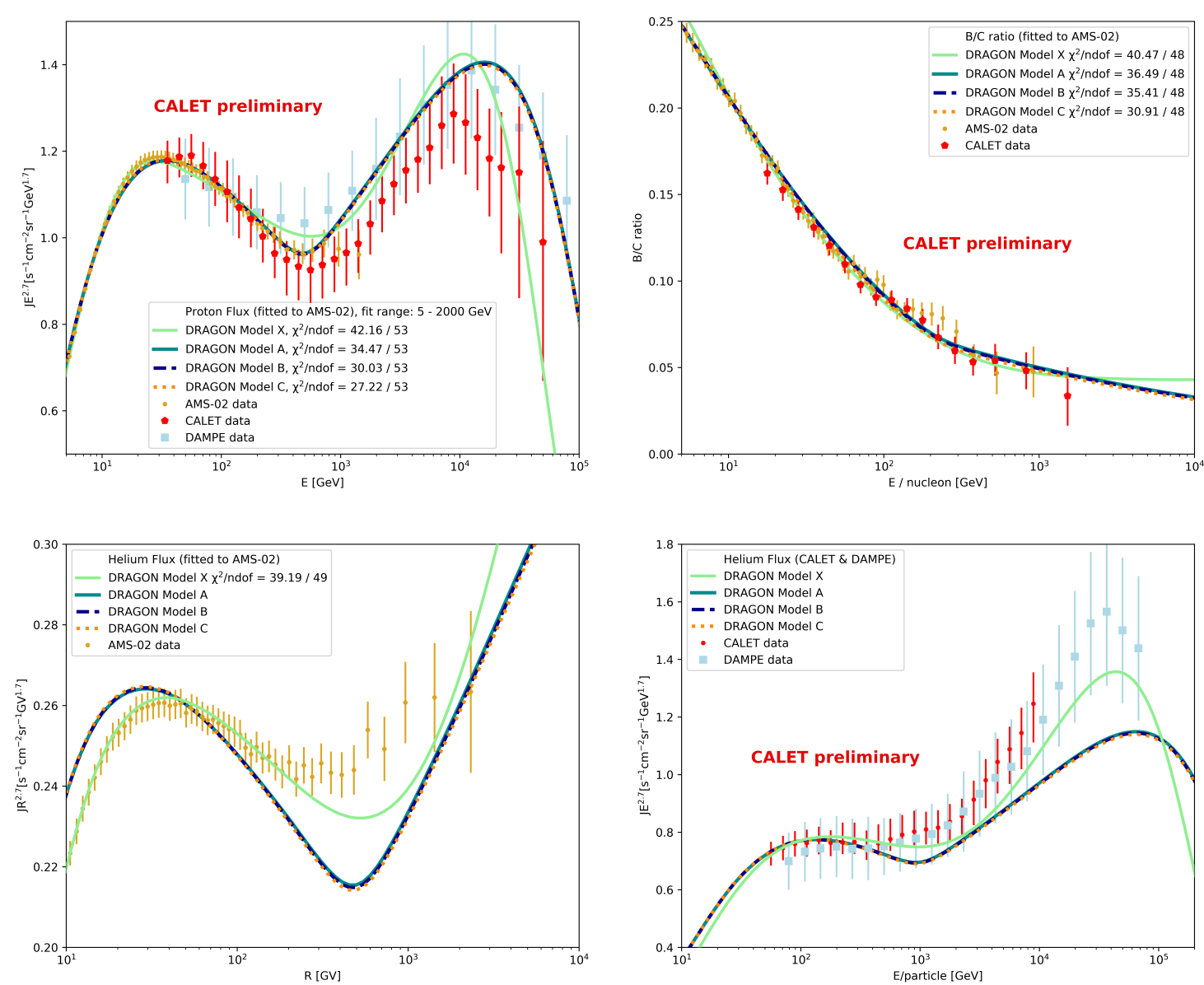

Figure 1: Comparison of the DRAGON calculation results to measurements. Top left: Proton spectrum fitted to AMS-02 data [6] and compared to CALET [7] and DAMPE [8] data. Top right: B/C ratio fitted to AMS-02 data [9] and compared to CALET [10] data. Bottom left: Helium spectrum in rigidity fitted (Model X) to AMS-02 data [11]. Bottom right: Helium spectrum in energy per particle compared to CALET [12] and DAMPE [13] data. For all experimental data the systematic errors are added quadratically to the statistical error.

The spectrum of primary electrons from distant SNR is parameterized by a power law with a soft spectral break (normalization $C_{e}$, index $\gamma_{e}$, break position $E_{b}$ and index change $\Delta \gamma_{e}$ are free fit parameters in the fit to CALET and AMS-02 data, softness $s=0.05$ is fixed) at low energy, and a high-energy exponential cut-off at $E_{c u t_{d}}=1 \mathrm{TeV}$ representing radiative energy loss of high energy electrons. The secondary positron $\left(\Phi_{s\left(e^{+}\right)}\right)$and electron $\left(\Phi_{s\left(e^{-}\right)}\right)$fluxes are taken from the nuclei DRAGON calculations with the scale factor $C_{\text {norm }}$ from normalization to the proton flux re-scaled by the free parameter $\left(C_{S} / C_{\text {norm }}\right)$ to account for remaining uncertainties in secondary particle production. For $\Phi_{\text {pulsars }}$, the pulsars within $1 \mathrm{kpc}$ and younger than $1 \mathrm{Myr}$ from the ATNF catalog [14] are considered. Their individual parameters (age, position, initial energy) are taken from the ATNF catalog, with the initial rotation energy calculated as $Q_{0}=\dot{E} T^{2} / \tau$, where $\tau=10 \mathrm{kyr}$ is the assumed spin-down timescale [15]. It is assumed that all pulsars share 
the same power-law with cut-off injection spectrum defined by spectral index, cut-off energy and acceleration efficiency, which are free parameters in the fitting. The spectrum injected by each pulsar is propagated using the analytic solution of the propagation equation for a point source as explained in Ref. [16]. The diffusion coefficient is approximated by the rigidity dependent function at the position solar system with normalization $D_{0(s o l)}$ listed in Table 1. $\Phi_{\text {near } S N R}$ is calculated with DRAGON as a point source at each SNR's position, with power law index $\gamma_{i}$ identical to that of the nucleons, and multiple values studied for the cut-off energy. Each SNR's injection spectrum is normalized to emitting $Q_{0_{S N R}}=10^{48}$ erg over $1 \mathrm{GeV}$ integrated over the injection duration, with the re-scaling factor varied in the fitting and limit calculation. Solar modulation is applied by the force field approximation with a constant modulation potential corresponding to the value at $10 \mathrm{GV}$ from the fit to the nuclei data for each propagation model listed in Table 1.

\subsection{Fitting and Limit Calculation}

The model is fitted to the data of CALET based on total flux $\Phi_{e}^{-}+\Phi_{e}^{+}$and data of AMS02 [3] for $E>10 \mathrm{GeV}$ based on $\Phi_{e}^{+}$by minimizing the sum of $\chi^{2}$ of both comparisons, with systematic uncertainties of both measurements taken into account. For the CALET measurement, the energy dependent $1 \sigma$ deviation $\Delta(E)$ is calculated for each data point in the same way as the values listed in the supplemental material of Ref. [17] for the following systematic uncertainty sources: Normalization, tracking, charge selection, electron identification, Monte Carlo model dependence. A shift by $w \Delta(E)$ is performed as part of the fit function with the weight $w$ for each systematic uncertainty source as a free parameter and each squared weight added to the total $\chi^{2}$ of the fit as explained in Ref. [16]. With this method, the known energy dependence of the systematic uncertainty connects the high energy range relevant for nearby SNR signatures to lower energy datapoints, improving the constraint on the model over quadratic addition of statistical and systematic error. Systematic errors without known energy dependence (trigger and BDT proton rejection) are added quadratically to the statistical error. For the AMS-02 measurement, the error on mean energy $\sigma_{E}$ in each bin is translated into an error on flux $\sigma_{J(E)}$ using the power law index $\gamma_{e^{+}}$also shown in Ref. [3] via the relation $\sigma_{J(E)}=J(E)\left(\sigma_{E} / E\right)\left(\gamma_{e^{+}}-1\right)$. To derive a limit on $Q_{0_{S N R}}$, the parameter is increased in steps until $\chi^{2}$ exceeds the $95 \%$ CL threshold, while all free parameters including the systematic uncertainty weights are re-adjusted to accommodate the increased flux from the nearby SNR. To determine the precise value of $Q_{0_{S N R}}$, for which the $95 \%$ CL threshold is crossed, the scan is repeated from the last allowed value with a factor 10 smaller step size, down to steps of $10^{45} \mathrm{erg}$.

\subsection{Comparison with Anisotropy Constraints}

For each calculated best-fit and limit-fit based on the spectrum, a test whether the anisotropy in this case is allowed by the Fermi-LAT anisotropy limits [18] or not is performed. The flux anisotropy for the nearby SNR is calculated with DRAGON using the method described in Ref [19], with the flux from distant SNR and the pulsars taken as isotropic. If the anisotropy exceeds Fermi-LAT limit, a best-fit model can be considered excluded, while in the case of the limit fit, it indicates that already a stronger limit based on the Fermi-LAT anisotropy measurement exists. 

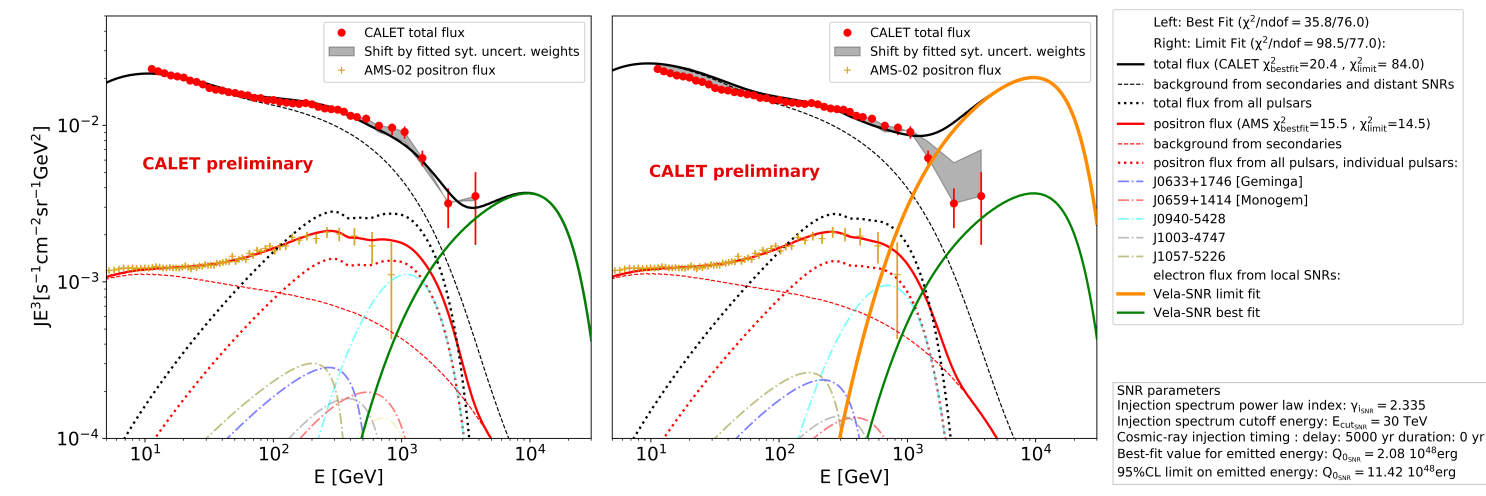

Figure 2: Best fit (left) and limit fit (right) for the case of $5 \mathrm{kyr}$ delay and $30 \mathrm{TeV}$ cut-off energy in propagation model X, including only Vela. See legend for explanation of each graph element. The weights of the systematic uncertainties in the best (limit) fit are $0.80(0.26)$ for normalization, 0.39 (4.09) for tracking, 0.28 (1.47) for charge selection, 1.75 (0.03) for electron identification, 0.34 (2.69) for Monte Carlo, shifting the reference for the fit function as indicated by the gray band while contributing 4.05 (26.2) to $\chi^{2}$.

\begin{tabular}{|c|c|c|c|c|c|c|c|c|c|}
\hline \multirow{3}{*}{ Model } & \multicolumn{7}{|c|}{ duration [kyr] } & \multicolumn{4}{c|}{ delay [kyr] } \\
\cline { 2 - 10 } & 0 & 1 & 2 & 5 & 10 & 1 & 2 & 5 & 10 \\
\cline { 2 - 10 } & \multicolumn{8}{|c|}{ Best-Fit $Q_{0}\left[10^{48} \mathrm{erg}\right]$} \\
\hline $\mathrm{X}$ & $0.37-0.58$ & $0.44-0.58$ & $0.43-0.65$ & $0.52-0.76$ & $0.93-1.33$ & $0.54-0.79$ & $0.47-0.67$ & $1.84-2.59$ & $2875-4037$ \\
$\mathrm{~A}$ & $0.35-0.49$ & $0.34-0.48$ & $0.33-0.48$ & $0.33-0.46$ & $0.42-0.59$ & $0.36-0.51$ & $0.32-0.46$ & $0.42-0.60$ & $5.68-8.10$ \\
$\mathrm{~B}$ & $0.55-0.78$ & $0.52-0.77$ & $0.50-0.71$ & $0.44-0.62$ & $0.37-0.53$ & $0.51-0.72$ & $0.45-0.65$ & $0.37-0.54$ & $0.55-0.77$ \\
$\mathrm{C}$ & $0.70-1.01$ & $0.92-1.01$ & $0.62-1.02$ & $0.57-0.83$ & $0.41-0.61$ & $0.58-0.92$ & $0.58-0.85$ & $0.43-0.65$ & $0.37-0.51$ \\
\cline { 2 - 10 } & \multicolumn{8}{|c|}{$95 \%$ CL-Limit $Q_{0}\left[10^{48} \mathrm{erg}\right]$} \\
$\mathrm{nyyyyyyyyy}$ X & $1.83-2.44$ & $1.92-2.56$ & $2.04-2.71$ & $2.63-3.47$ & $4.62-6.10$ & $2.59-3.41$ & $2.35-3.10$ & $10.6-13.9$ & $21365-28612$ \\
$\mathrm{~A}$ & $1.40-1.75$ & $1.39-1.74$ & $1.38-1.74$ & $1.40-1.79$ & $1.87-2.43$ & $1.50-1.91$ & $1.37-1.73$ & $2.00-2.62$ & $41.3-56.2$ \\
$\mathrm{~B}$ & $2.06-2.48$ & $1.98-2.38$ & $1.90-2.29$ & $1.68-2.05$ & $1.60-2.00$ & $1.92-2.34$ & $1.75-2.12$ & $1.57-1.94$ & $3.19-4.17$ \\
$\mathrm{C}$ & $2.73-3.27$ & $2.60-3.12$ & $2.48-2.97$ & $2.11-2.54$ & $1.73-2.13$ & $2.47-2.97$ & $2.25-2.70$ & $1.74-2.11$ & $1.88-2.42$ \\
\hline
\end{tabular}

Table 2: Result overview for the Vela-only study. Each cell shows the range of $Q_{0_{S N R}}$ with variation of the cut-off energy, where in general the higher value is for $10 \mathrm{TeV}$ and the lower for $200 \mathrm{TeV}$.

\section{Results}

\subsection{Vela-only Best-fit and Limit}

For each propagation model, the two cases of continuous cosmic ray injection with constant intensity and of delayed burst-like injection have been studied for injection duration or delay of 0,1 , 2, 5 and $10 \mathrm{kyr}$ respectively. For each model and duration/delay time, the values of 10, 20, 30, 50, 100 and $200 \mathrm{TeV}$ were studied for the source spectrum cut-off energy, with all fits yielding reduced $\chi^{2}$ around one half, so that no significant preference for these parameters can be derived from the fit. Figure 2 shows the best and limit fit spectra for the example case of $5 \mathrm{kyr}$ delayed injection and $30 \mathrm{TeV}$ source cut-off energy in Model X. Table 2 gives an overview of the obtained best-fit and limit values for $Q_{0_{S N R}}$, indicating that the variation of either is within a factor four, except for the cases with low diffusion speed and late release of the cosmic rays (Model X, $5 \mathrm{kyr}$ and $10 \mathrm{kyr}$ delay; Model A, 10 kyr delay), for which only the highest energy electrons arrive within the short 

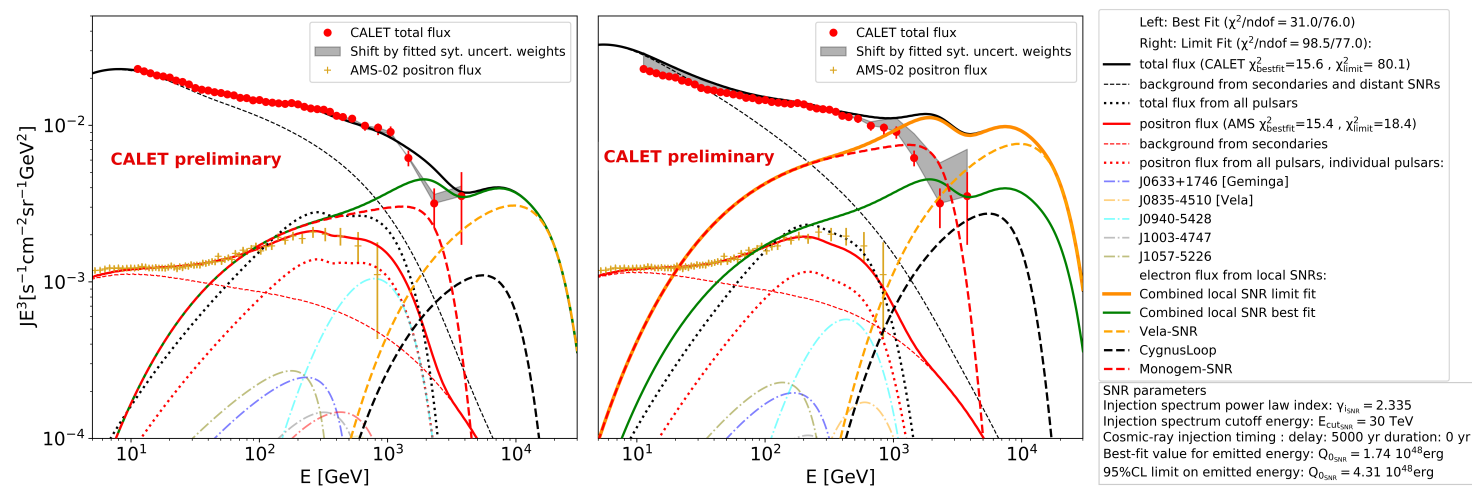

Figure 3: Best fit (left) and limit fit (right) for the case of $5 \mathrm{kyr}$ delay and $30 \mathrm{TeV}$ cut-off energy, including all three nearby SNR in propagation model X. See legend for explanation of each graph element. The fitted weights of the systematic uncertainties in the best (limit) fit are 0.68 (2.70) for normalization, 0.91 (2.57) for tracking, 0.70 (1.57) for charge selection, 0.39 (2.38) for electron identification, 0.17 (2.63) for Monte Carlo, shifting the reference for the fit function as indicated by the gray band while contributing $1.96(28.9)$ to $\chi^{2}$.

\begin{tabular}{|c|c|c|c|c|c|c|c|c|c|}
\hline \multirow{3}{*}{ Model } & \multicolumn{5}{|c|}{ duration [kyr] } & \multicolumn{4}{|c|}{ delay $[\mathrm{kyr}]$} \\
\hline & 0 & 1 & 2 & 5 & 10 & 1 & 2 & 5 & 10 \\
\hline & \multicolumn{9}{|c|}{ Best-Fit $Q_{0}\left[10^{48} \mathrm{erg}\right]$} \\
\hline $\mathrm{X}$ & $0.33-0.50$ & $0.35-0.57$ & $0.36-0.55$ & $0.44-0.71$ & $0.70-1.21$ & $0.43-0.70$ & $0.41-0.67$ & $1.38-2.23$ & $2.05-3.17$ \\
\hline A & $0.27-0.40$ & $0.27-0.37$ & $0.26-0.39$ & $0.25-0.37$ & $0.31-0.45$ & $0.28-0.41$ & $0.25-0.38$ & $0.31-0.45$ & $1.08-1.70$ \\
\hline B & $0.40-0.61$ & $0.40-0.59$ & $0.38-0.57$ & $0.34-0.50$ & $0.29-0.45$ & $0.39-0.56$ & $0.35-0.51$ & $0.30-0.44$ & $0.39-0.58$ \\
\hline \multirow[t]{2}{*}{$\mathrm{C}$} & $0.52-0.81$ & $0.52-0.86$ & $0.50-0.73$ & $0.43-0.66$ & $0.33-0.50$ & $0.51-0.71$ & $0.43-0.68$ & $0.35-0.53$ & $0.29-0.40$ \\
\hline & \multicolumn{9}{|c|}{$95 \%$ CL-Limit $Q_{0}\left[10^{48} \mathrm{erg}\right]$} \\
\hline $\mathrm{X}$ & $1.38-1.89$ & $1.44-1.97$ & $1.52-2.09$ & $1.85-2.55$ & $2.67-3.58$ & $1.85-2.55$ & $1.70-2.34$ & $3.94-5.05$ & $4.82-5.63$ \\
\hline A & $1.32-1.05$ & $1.05-1.33$ & $1.05-1.33$ & $1.07-1.38$ & $1.34-1.77$ & $1.12-1.44$ & $1.03-1.32$ & $1.41-1.89$ & $4.62-6.43$ \\
\hline B & $1.48-1.78$ & $1.45-1.75$ & $1.40-1.70$ & $1.26-1.55$ & $1.20-1.50$ & $1.40-1.70$ & $1.29-1.56$ & $1.17-1.45$ & $1.83-2.44$ \\
\hline $\mathrm{C}$ & $1.91-2.30$ & $1.89-2.26$ & $1.81-2.17$ & $1.58-1.90$ & $1.33-1.63$ & $1.78-2.13$ & $1.64-1.96$ & $1.32-1.59$ & $1.34-1.75$ \\
\hline
\end{tabular}

Table 3: Result overview for the study combining Vela, Monogem and Cynus Loop signatures. Each cell shows the range of $Q_{0_{S N R}}$ with variation of the cut-off energy, where in general the higher value is for $10 \mathrm{TeV}$ and the lower for $200 \mathrm{TeV}$.

diffusion time, giving limits larger by orders of magnitude. For Model X and A, all best-fit cases are allowed by the Fermi-LAT anisotropy limit, while for Model $\mathrm{C}$ all cases are excluded. For Model B, some cases with 100 and 200 TV cut-off energy and release within 2 kyr are allowed. For all cases considering only Vela, a more stringent limit from anisotropy exists.

\subsection{Nearby SNR Best-fit and Limit}

The fit and limit calculation has also been performed including the signatures of the three significantly contributing nearby SNR, namely Vela, Monogem and Cygnus Loop. Except age and position, the three SNR are assumed to have identical properties, including $Q_{0_{S N R}}$. Figure 3 shows the best and limit fit spectra for the example case of $5 \mathrm{kyr}$ delayed injection and $30 \mathrm{TeV}$ source cut-off energy in Model X. An overview of the results is given in Table 3. The contribution of Monogem and Cygnus Loop reduces the values compared to the Vela-only scenario, especially 
for the aforementioned low-diffusion, late-release cases. Limits ranging from $3.94 \times 10^{48} \mathrm{erg}$ to $6.43 \times 10^{48} \mathrm{erg}$ can be set for the Model X, $5 \mathrm{kyr}$ and $10 \mathrm{kyr}$ delay, Model A, $10 \mathrm{kyr}$ delay cases. These are also the cases for which the limit fit is not excluded by anisotropy, i.e. these limits are stricter than those from Fermi-LAT. Among the best-fit cases, all of Models X and A are allowed by the anisotropy limit, while for Model B most cases and for Model C only a few with high cut-off energy and early release pass this constraint.

\section{Conclusion}

From fitting the DRAGON-calculated flux of nearby SNR to the high-energy CALET allelectron spectrum, the preferred range for the energy emitted by a SNR in electron cosmic rays is found to be in a range of several $10^{47}$ erg over a wide variety of conditions for propagation, release timing and spectral cut-off, with $95 \% \mathrm{CL}$ limits on the order of a few $10^{48} \mathrm{erg}$. Conditions under which cosmic rays from Vela can only partly propagate to Earth due to delayed release and low propagation speed constitute a special case where no strong constraint can be set on the Vela SNR alone, but limits of $\sim 5 \times 10^{48} \mathrm{erg}$, exceeding those from Fermi-LAT anisotropy data, can be set, if considering the combined flux from all the nearby SNR: Vela, Monogem and Cygnus Loop.

\section{Acknowledgements}

This work was supported by JSPS KAKENHI Grant Number JP21K03604.

\section{References}

[1] T. Kobayashi, Y. Komori, K. Yoshida, J. Nishimura, Astrophys. J. 601, 340 (2004).

[2] S. Torii, et al., PoS ICRC2021 (2021). In press.

[3] M. Aguilar, et al., Phys. Rev. Lett. 122, 041102 (2019).

[4] D. Gaggero, et al., Phys.Rev.Lett. 111, 021102 (2013).

[5] I. Cholis, D. Hooper, T. Linden, Phys. Rev. D 93, 043016 (2016).

[6] M. Aguilar, et al., Phys. Rev. Lett. 114, 171103 (2015).

[7] K. Kobayashi, et al., PoS ICRC2021 (2021). In press.

[8] Q. An, et al., Sci. Adv. 5, eaax3793 (2019).

[9] M. Aguilar, et al., Phys. Rev. Lett. 117, 231102 (2016).

[10] Y. Akaike, et al., PoS ICRC2021 (2021). In press.

[11] M. Aguilar, et al., Phys. Rev. Lett. 115, 211101 (2015).

[12] P. Brogi, et al., PoS ICRC2021 (2021). In press.

[13] F. Alemanno, et al., Phys. Rev. Lett. 126, 201102 (2021).

[14] R. N. Manchester, G. B. Hobbs, A. Teoh, M. Hobbs, Astron.J. 129, 1993 (2005).

[15] J. Feng, H.-H. Zhang, Eur. Phys. J. C 76, 229 (2016).

[16] H. Motz, Y. Asaoka, S. Bhattacharyya, PoS ICRC2019, 533 (2019).

[17] O. Adriani, et al., Phys. Rev. Lett. 120, 261102 (2018).

[18] S. Abdollahi, et al., Phys. Rev. Lett. 118, 091103 (2017).

[19] H. Motz, Y. Asaoka, et al., PoS ICRC2019, 358 (2019). 


\section{Full Authors List: CALET Collaboration}

O. Adriani ${ }^{1,2}$, Y. Akaike ${ }^{3,4}$, K. Asano ${ }^{5}$, Y. Asaoka ${ }^{5}$, E. Berti 1,2 , G. Bigongiari 6,7 , W. R. Binns ${ }^{8}$, M. Bongi ${ }^{1,2}$, P. Brogi ${ }^{6,7}$, A. Bruno ${ }^{9,10}$, J. H. Buckley ${ }^{8}$, N. Cannady ${ }^{11,12,13}$, G. Castellini ${ }^{14}$, C. Checchia ${ }^{6}$, M. L. Cherry ${ }^{15}$, G. Collazuol ${ }^{16,17}$, K. Ebisawa ${ }^{18}$, A. W. Ficklin ${ }^{15}$, H. Fuke ${ }^{18}$, S. Gonzi ${ }^{1,2}$, T. G. Guzik ${ }^{15}$, T. Hams ${ }^{11}$, K. Hibino ${ }^{19}$, M. Ichimura ${ }^{20}$, K. Ioka $^{21}$, W. Ishizaki ${ }^{5}$, M. H. Israel ${ }^{8}$, K. Kasahara ${ }^{22}$, J. Kataoka ${ }^{23}$, R. Kataoka ${ }^{24}$, Y. Katayose ${ }^{25}$,

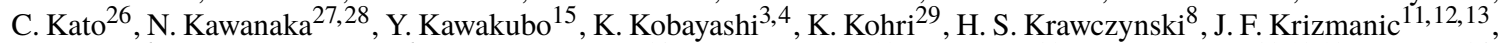
P. Maestro $^{6,7}$, P. S. Marrocchesi 6,7 , A. M. Messineo ${ }^{30,7}$, J.W. Mitchell ${ }^{12}$, S. Miyake ${ }^{32}$, A. A. Moiseev ${ }^{33,12,13}$, M. Mori ${ }^{34}$, N. Mori ${ }^{2}$, H. M. Motz ${ }^{35}$, K. Munakata ${ }^{26}$, S. Nakahira ${ }^{18}$, J. Nishimura ${ }^{18}$, G. A. de Nolfo ${ }^{9}$, S. Okuno ${ }^{19}$, J. F. Ormes ${ }^{36}$, N. Ospina ${ }^{16,17}$, S. Ozawa ${ }^{37}$, L. Pacini ${ }^{1,14,2}$, P. Papini ${ }^{2}$, B. F. Rauch ${ }^{8}$, S. B. Ricciarini ${ }^{14,2}$, K. Sakai ${ }^{11,12,13}$, T. Sakamoto ${ }^{38}$, M. Sasaki ${ }^{33,12,13}$, Y. Shimizu ${ }^{19}$, A. Shiomi ${ }^{39}$, P. Spillantini ${ }^{1}$, F. Stolzi 6,7 , S. Sugita ${ }^{38}$, A. Sulaj 6,7 , M. Takita ${ }^{5}$, T. Tamura ${ }^{19}$, T. Terasawa ${ }^{40}$, S. Torii ${ }^{3}$, Y. Tsunesada ${ }^{41}$, Y. Uchihori ${ }^{42}$, E. Vannuccini ${ }^{2}$, J. P. Wefel ${ }^{15}$, K. Yamaoka ${ }^{43}$, S. Yanagita ${ }^{44}$, A. Yoshida ${ }^{38}$, K. Yoshida ${ }^{22}$, and W. V. Zober ${ }^{8}$

${ }^{1}$ Department of Physics, University of Florence, Via Sansone, 1, 50019 Sesto, Fiorentino, Italy, ${ }^{2}$ INFN Sezione di Florence, Via Sansone, 1, 50019 Sesto, Fiorentino, Italy, ${ }^{3}$ Waseda Research Institute for Science and Engineering, Waseda University, 17 Kikuicho, Shinjuku, Tokyo 162-0044, Japan, ${ }^{4}$ JEM Utilization Center, Human Spaceflight Technology Directorate, Japan Aerospace Exploration Agency, 2-1-1 Sengen, Tsukuba, Ibaraki 305-8505, Japan, ${ }^{5}$ Institute for Cosmic Ray Research, The University of Tokyo, 5-1-5 Kashiwa-no-Ha, Kashiwa, Chiba 277-8582, Japan, ${ }^{6}$ Department of Physical Sciences, Earth and Environment, University of Siena, via Roma 56, 53100 Siena, Italy, ${ }^{7}$ INFN Sezione di Pisa, Polo Fibonacci, Largo B. Pontecorvo, 3, 56127 Pisa, Italy, ${ }^{8}$ Department of Physics and McDonnell Center for the Space Sciences, Washington University, One Brookings Drive, St. Louis, Missouri 63130-4899, USA, ${ }^{9}$ Heliospheric Physics Laboratory, NASA/GSFC, Greenbelt, Maryland 20771, USA, ${ }^{10}$ Department of Physics, Catholic University of America, Washington, DC 20064, USA, ${ }^{11}$ Center for Space Sciences and Technology, University of Maryland, Baltimore County, 1000 Hilltop Circle, Baltimore, Maryland 21250, USA, ${ }^{12}$ Astroparticle Physics Laboratory, NASA/GSFC, Greenbelt, Maryland 20771, USA, ${ }^{13}$ Center for Research and Exploration in Space Sciences and Technology, NASA/GSFC, Greenbelt, Maryland 20771, USA, ${ }^{14}$ Institute of Applied Physics (IFAC), National Research Council (CNR), Via Madonna del Piano, 10, 50019 Sesto, Fiorentino, Italy, ${ }^{15}$ Department of Physics and Astronomy, Louisiana State University, 202 Nicholson Hall, Baton Rouge, Louisiana 70803, USA, ${ }^{16}$ Department of Physics and Astronomy, University of Padova, Via Marzolo, 8, 35131 Padova, Italy, ${ }^{17}$ INFN Sezione di Padova, Via Marzolo, 8, 35131 Padova, Italy, ${ }^{18}$ Institute of Space and Astronautical Science, Japan Aerospace Exploration Agency, 3-1-1 Yoshinodai, Chuo, Sagamihara, Kanagawa 252-5210, Japan, ${ }^{19}$ Kanagawa University, 3-27-1 Rokkakubashi, Kanagawa, Yokohama, Kanagawa 221-8686, Japan, ${ }^{20}$ Faculty of Science and Technology, Graduate School of Science and Technology,, Hirosaki University, 3, Bunkyo, Hirosaki, Aomori 036-8561, Japan, ${ }^{21}$ Yukawa Institute for Theoretical Physics, Kyoto University, Kitashirakawa Oiwakecho, Sakyo, Kyoto 606-8502, Japan, ${ }^{22}$ Department of Electronic Information Systems, Shibaura Institute of Technology, 307 Fukasaku, Minuma, Saitama 337-8570, Japan, ${ }^{23}$ School of Advanced Science and Engineering, Waseda University, 3-4-1 Okubo, Shinjuku, Tokyo 169-8555, Japan, ${ }^{24}$ National Institute of Polar Research, 10-3, Midori-cho, Tachikawa, Tokyo 190-8518, Japan, ${ }^{25}$ Faculty of Engineering, Division of Intelligent Systems Engineering, Yokohama National University, 79-5 Tokiwadai, Hodogaya, Yokohama 240-8501, Japan, ${ }^{26}$ Faculty of Science, Shinshu University, 3-1-1 Asahi, Matsumoto, Nagano 390-8621, Japan, ${ }^{27}$ Hakubi Center, Kyoto University, Yoshida Honmachi, Sakyo-ku, Kyoto 606-8501, Japan, ${ }^{28}$ Department of Astronomy, Graduate School of Science, Kyoto University, Kitashirakawa Oiwake-cho, Sakyo-ku, Kyoto 6068502, Japan, ${ }^{29}$ Institute of Particle and Nuclear Studies, High Energy Accelerator Research Organization, 1-1 Oho, Tsukuba, Ibaraki 305-0801, Japan, ${ }^{30}$ University of Pisa, Polo Fibonacci, Largo B. Pontecorvo, 3, 56127 Pisa, Italy, ${ }^{31}$ Astroparticle Physics Laboratory, NASA/GSFC, Greenbelt, Maryland 20771, USA, ${ }^{32}$ Department of Electrical and Electronic Systems Engineering, National Institute of Technology, Ibaraki College, 866 Nakane, Hitachinaka, Ibaraki 312-8508, Japan ${ }^{33}$ Department of Astronomy, University of Maryland, College Park, Maryland 20742, USA, ${ }^{34}$ Department of Physical Sciences, College of Science and Engineering, Ritsumeikan University, Shiga 525-8577, Japan, ${ }^{35}$ Faculty of Science and Engineering, Global Center for Science and Engineering, Waseda University, 3-4-1 Okubo, Shinjuku, Tokyo 169-8555, Japan, ${ }^{36}$ Department of Physics and Astronomy, University of Denver, Physics Building, Room 211, 2112 East Wesley Avenue, Denver, Colorado 80208-6900, USA, ${ }^{37}$ Quantum ICT Advanced Development Center, National Institute of Information and Communications Technology, 4-2-1 Nukui-Kitamachi, Koganei, Tokyo 184-8795, Japan, ${ }^{38}$ College of Science and Engineering, Department of Physics and Mathematics, Aoyama Gakuin University, 5-10-1 Fuchinobe, Chuo, Sagamihara, Kanagawa 252-5258, Japan, ${ }^{39}$ College of Industrial Technology, Nihon University, 1-2-1 Izumi, Narashino, Chiba 275-8575, Japan ${ }^{40}$ RIKEN, 2-1 Hirosawa, Wako, Saitama 351-0198, Japan, ${ }^{41}$ Division of Mathematics and Physics, Graduate School of Science, Osaka City University, 3-3-138 Sugimoto, Sumiyoshi, Osaka 558-8585, Japan, ${ }^{42}$ National Institutes for Quantum and Radiation Science and Technology, 4-9-1 Anagawa, Inage, Chiba 263-8555, Japan, ${ }^{43}$ Nagoya University, Furo, Chikusa, Nagoya 464-8601, Japan, ${ }^{44}$ College of Science, Ibaraki University, 2-1-1 Bunkyo, Mito, Ibaraki 310-8512, Japan 